\title{
Lung cancer exosomes initiate global long non-coding RNA changes in mesenchymal stem cells
}

\author{
SHIHUA WANG ${ }^{*}$, XIAOXIA LI ${ }^{*}$, RONGJIA ZHU, QIN HAN and ROBERT CHUNHUA ZHAO \\ Institute of Basic Medical Sciences Chinese Academy of Medical Sciences, School of Basic Medicine \\ Peking Union Medical College, Peking Union Medical College Hospital, Center of Excellence in \\ Tissue Engineering Chinese Academy of Medical Sciences, Beijing 100005, P.R. China
}

Received October 19, 2015; Accepted November 11, 2015

DOI: $10.3892 /$ ijo.2015.3272

\begin{abstract}
Mesenchymal stem cells (MSCs) can be attracted to tumor sites and become an important component of the tumor microenvironment, thus contributing to tumor development. Emerging evidence suggests that tumor cells could transfer genetic information into MSCs through the release of exosomes. However, the molecular mechanisms by which tumor exosomes contribute to interactions between MSCs and tumor cells remain largely unknown. In this study, we found that lung tumor cell derived exosomes could inhibit MSCs osteogenic and adipogenic differentiation. We then investigated the involvement of long non-coding RNAs, a new class of regulators, in tumor exosome treated MSCs by a comprehensive lncRNA and mRNA profiling. IncRNAs (9.1\%) (2775 out of 30586) and 9.3\% of protein-coding mRNA (2439 out of 26109) were differentially expressed (fold-change $\geq 2$; P-value $\leq 0.05$ ) in lung tumor cell exosome treated MSCs. Furthermore, we characterized the differentially expressed lncRNAs through their classes and length distribution and correlated them with differentially expressed mRNA. Noteworthy, GO analysis of biological process showed that upregulated mRNAs were enriched in mRNA metabolic process, while downregulated ones were enriched in detection of mechanical stimulus involved in sensory perception. Pathway analysis indicated that 32 pathways were upregulated while 7 were downregulated in A549 exosome treated MSCs.
\end{abstract}

Correspondence to: Dr Qin Han or Professor Robert Chunhua Zhao, Institute of Basic Medical Sciences Chinese Academy of Medical Sciences, School of Basic Medicine Peking Union Medical College, Peking Union Medical College Hospital, Center of Excellence in Tissue Engineering Chinese Academy of Medical Sciences, 5 Dongdan santiao, Beijing 100005, P.R. China

E-mail: hanqinhanqin@126.com

E-mail: zhaochunhua@vip.163.com

*Contributed equally

Key words: lung tumor, A549 cell line, exosome, long non-coding RNA, mesenchymal stem cell
Here, we are the first to determine genome-wide lncRNA expression patterns in exosome treated MSCs by microarray and the results will bring new insights into the mechanisms underlying interactions between tumor cells exosomes and its environmental component the MSCs.

\section{Introduction}

Solid tumors are composed of not only tumor cells but also a complex array of stromal cells (1). Accumulating evidence has demonstrated that tumor-associated stromal cells play important roles in providing support for tumor cells, thus contributing to tumor initiation and progression $(2,3)$. One frequent component of the tumor stroma is tumor-associated fibroblasts (TAFs) that express $\alpha$-smooth muscle actin $(\alpha-$ SMA $)$ and has been reported to exert fundamental effects on tumor progression (4). Tumors could recruit mesenchymal stem cells (MSCs) into their microenvironment, where they become TAFs and affect tumor cell survival, angiogenesis and metastasis $(5,6)$. MSCs are defined as multipotent stem cells that have the capacity to give rise to adipocytes, osteoblasts and chondrocytes (7). MSCs can be isolated from a number of tissues including bone marrow, adipose tissue and umbilical cord blood. Several studies have pointed to MSCs as an important source of TAFs $(1,8)$. The precise role of MSCs in tumor initiation and progression is still controversial because both pro- and anti-tumorigenic effects have been reported.

Interactions between tumor cells and MSCs within the local microenvironment could be mediated by cell-cell contact and by paracrine mechanisms through release of a variety of bioactive molecules such as growth factors, cytokines and inflammatory mediators $(9,10)$. Recently, a novel way emerged of cell-cell communication mediated through exosomes, which are small membrane vesicles secreted by a variety of cell types, including tumor cells. Numerous reports showed that tumor exosomes are associated with tumor development, chemoresistance and capacity to escape from immune surveillance (11-13). Although the biological functions are not well-defined, exosomes are known to deliver diverse molecules to target cells ranging from mRNAs, miRNAs, to proteins. Recent studies demonstrated that tumor-derived exosomes can function in communication between tumor cells and MSCs in the neoplastic tumor microenvironment (14-16). However, the 
precise mechanisms underlying interactions between MSCs and tumor exosomes remain largely unknown. Unraveling these mechanisms is of great significance because they may lead to novel preventive or therapeutic paradigms.

In this study, we explored the potential involvement of long non-coding RNAs (lncRNAs) in crosstalk between lung tumor cell derived exosomes and MSCs. We performed a comprehensive IncRNA and mRNA profiling through microarray. IncRNAs (9.1\%) (2775 out of 30586) and $9.3 \%$ of protein-coding mRNA (2439 out of 26109) were differentially expressed (fold-change $\geq 2$; P-value $\leq 0.05$ ) in lung tumor derived exosome stimulated MSCs. Furthermore, we characterized the differentially expressed lncRNAs through their classes and length distribution and correlated them with differentially expressed mRNA. Of note, GO analysis of biological process showed that upregulated mRNAs were enriched in mRNA metabolic process, while downregulated ones were enriched in detection of mechanical stimulus involved in sensory perception. Pathway analysis indicated that 32 pathways were upregulated while 7 were downregulated in A549 exosome treated MSCs. To the best of our knowledge, this is the first study that gives a comprehensive overview of the lncRNA transcriptome changes in MSCs after stimulation with tumor derived exosomes, which will bring new insights into the mechanisms underlying interactions between tumor cells exosomes and its environmental component the MSCs.

\section{Materials and methods}

Cell culture. Adipose tissues were obtained from patients undergoing liposuction according to procedures approved by the Ethics Committee at the Chinese Academy of Medical Sciences and Peking Union Medical College. MSCs were isolated and culture-expanded as previously reported (17). Passage 3 cells were used for following experiments. Lung cancer cell line A549 was purchased from cell bank at the Chinese Academy of Medical Sciences. A549 cells were cultured in DF12 containing 10\% fetal bovine serum (FBS), penicillin $(100 \mathrm{U} / \mathrm{ml})$ and streptomycin $(100 \mathrm{lg} / \mathrm{ml})$ at $37^{\circ} \mathrm{C}$ in humidified air with $5 \% \mathrm{CO}_{2}$.

Exosome extraction. Exosome extraction was performed as previously described (18). Briefly, A549 culture medium was collected and centrifuged at $800 \mathrm{x} \mathrm{g}$ for $5 \mathrm{~min}$ and additional $2,000 \mathrm{x} \mathrm{g}$ for $10 \mathrm{~min}$ to remove lifted cells. The supernatant was subjected to filtration on a $0.1-\mathrm{mm}$ pore polyethersulfone membrane filter (Corning) to remove cell debris and large vesicles, followed by concentration by a 100,000 MW cut-off membrane (CentriPlus-70, Millipore). The volume of supernatant was reduced from approximately $250-500 \mathrm{ml}$ to approximately $30 \mathrm{ml}$. The supernatant was then ultracentrifuged at $100,000 \mathrm{x}$ g for $1 \mathrm{~h}$ at $4^{\circ} \mathrm{C}$ using $70 \mathrm{Ti}$ rotor (Beckman Coulter). The resulting pellets were resuspended in $6 \mathrm{ml} \mathrm{PBS}$ and ultracentrifuged at $100,000 \mathrm{x} \mathrm{g}$ for $1 \mathrm{~h}$ at $4^{\circ} \mathrm{C}$ using 100Ti rotor (Beckman Coulter).

PKH67-labeled exosomes to AD-MSCs. Purified A549exosomes were labeled with $1 \mu \mathrm{M}$ Dil (Invitrogen) as previously described (19). Pelleted exosomes were washed to remove unbound $\mathrm{PKH} 67$, resuspended in $\mathrm{PBS} / 5 \% \mathrm{BSA}$ and then added to AD-MSC medium for $4 \mathrm{~h}$. AD-MSCs were then washed in PBS, fixed in $4 \%$ paraformaldehyde, and imaged by microscopy.

Transmission electron microscopy. Purified exosomes were fixed with $1 \%$ glutaraldehyde in PBS ( $\mathrm{pH}$ 7.4). After rinsing, a $20 \mu \mathrm{l}$ drop of the suspension was loaded onto a formvar/carbon-coated grid, negatively stained with $3 \%(\mathrm{w} / \mathrm{v})$ aqueous phosphotungstic acid for $1 \mathrm{~min}$, and observed by transmission electron microscopy.

Microarray and data analysis. Microarray and data analysis were performed by KangChen Biotechnology, Shanghai, China. The company used Arraystar Human LncRNA Microarray V3.0 which is designed for the global profiling of human lncRNAs and protein-coding transcripts. Approximately 30,586 lncRNAs and 26,109 coding transcripts can be detected by this third-generation lncRNA microarray.

i) RNA labeling and array hybridization: sample labeling and array hybridization were performed according to the Agilent One-Color Microarray-Based Gene Expression Analysis protocol (Agilent Technology) with minor modifications. Briefly, mRNA was purified from total RNA after removal of rRNA (mRNA-ONLY ${ }^{\mathrm{TM}}$ Eukaryotic mRNA Isolation kit, Epicentre). Then, each sample was amplified and transcribed into fluorescent cRNA along the entire length of the transcripts without $3^{\prime}$ bias utilizing a random priming method (Arraystar Flash RNA Labeling kit, Arraystar).

The labeled cRNAs were purified by RNeasy Mini kit (Qiagen). The concentration and specific activity of the labeled cRNAs (pmol Cy3/ $\mu$ g cRNA) were measured by NanoDrop ND-1000. One microgram of each labeled cRNA was fragmented by adding $5 \mu \mathrm{l} 10 \mathrm{X}$ Blocking Agent and $1 \mu \mathrm{l}$ of 25X Fragmentation Buffer, then heated the mixture at $60^{\circ} \mathrm{C}$ for $30 \mathrm{~min}$, finally $25 \mu \mathrm{l} 2 \mathrm{X}$ GE Hybridization buffer was added to dilute the labeled cRNA. Hybridization solution $(50 \mu \mathrm{l})$ was dispensed into the gasket slide and assembled to the LncRNA expression microarray slide. The slides were incubated for $17 \mathrm{~h}$ at $65^{\circ} \mathrm{C}$ in an Agilent Hybridization Oven. The hybridized arrays were washed, fixed and scanned with using the Agilent DNA Microarray Scanner (part number G2505C).

ii) Data analysis: slides were scanned at $5 \mathrm{~lm} /$ pixel resolution using an Axon GenePix 4000B scanner (Molecular Devices Corp.) piloted by GenePix Pro 6.0 software (Axon). Scanned images (TIFF format) were then imported into NimbleScan software (version 2.5) for grid alignment and expression data analysis. Expression data were normalized through quantile normalization and the Robust Multichip Average (RMA) algorithm included in the NimbleScan software. The probe level files and mRNA level files were generated after normalization. All gene level files were imported into Agilent GeneSpring GX software (version 11.5.1) and normalized by the quantile method; then, Combat software was used to adjust the normalized intensity to remove batch effects. Hierarchical clustering was performed using Agilent GeneSpring GX software (version 11.5.1). Agilent Feature Extraction software (version 11.0.1.1) was used to analyze acquired array images. 
A

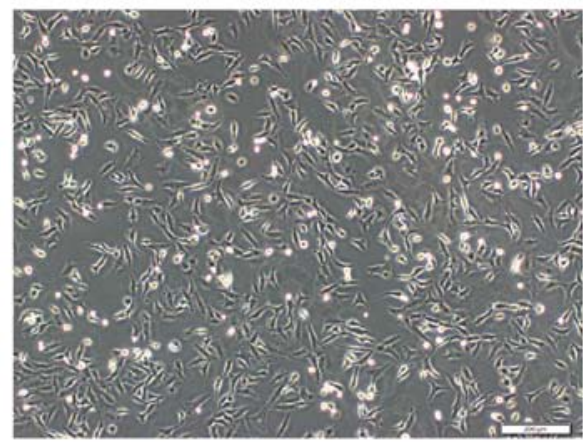

B

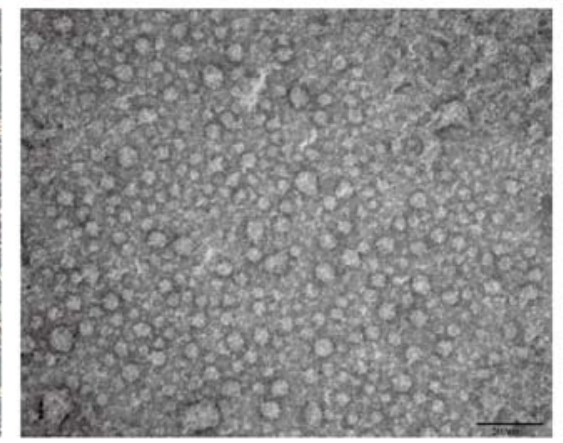

C

Cells Exosome

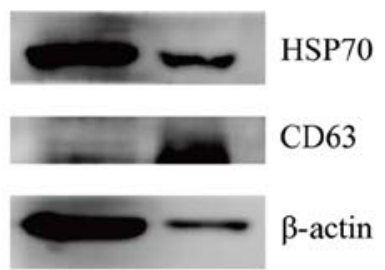

D

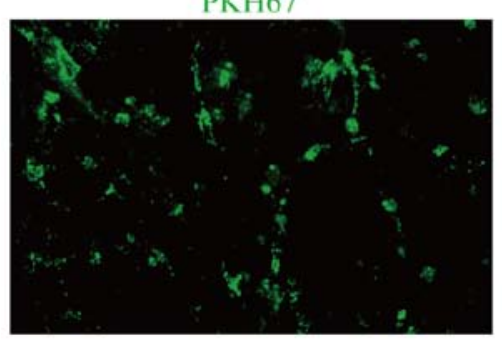

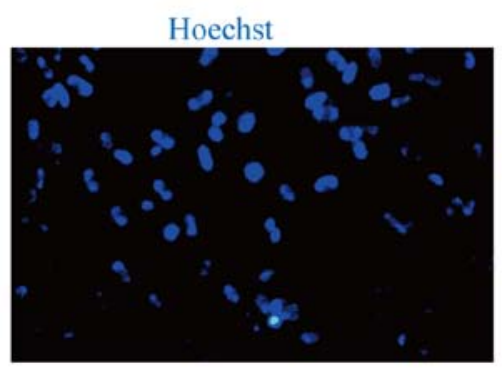

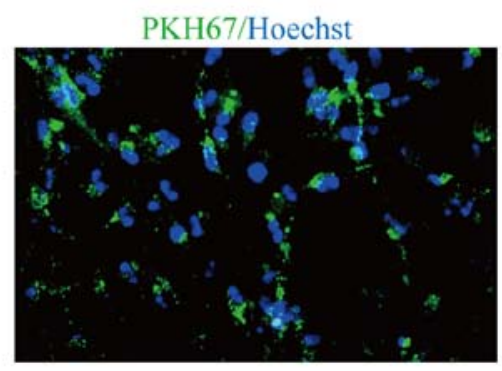

Figure 1. Characterization of exosomes secreted by lung tumor cells A549. (A) The cell morphology of A549 was observed under light microscope. (B) Electron micrograph of exosomes from A549. Scale bar, $200 \mathrm{~nm}$. (C) Detection of CD63 and HSP70 expression in A549-exosomes by western blotting. (D) PKH67-labeled A549 exosomes can label human AD-MSCs.

\section{Results}

Characterization of lung tumor cell A549-derived exosomes. Exosomes were isolated from the culture supernatants of lung tumor cell line A549 through a series of centrifugation and filtration steps. Fig. 1A shows the morphology of A549 cells under a light microscope. The exosome concentration in A549 culture medium was $21.2 \pm 3.2 \mu \mathrm{g} / \mathrm{ml}$. Under transmission electron microscopy, the exosomes were observed to be round vesicles of approximately 30-100 $\mathrm{nm}$ in size (Fig. 1B). CD63 and HSP70, typical protein markers of exosomes, were detectable in the A549-derived exosomes (Fig. 1C). To examine whether A549-exosomes could be transferred into AD-MSCs, the exosomes were fluorescently labeled with PKH67 and incubated with MSCs for $4 \mathrm{~h}$. After treatment, over $80 \%$ of the MSCs cells exhibited green fluorescence (Fig. 1D).

A549-derived exosomes inhibited osteogenic and adipogenic differentiation of AD-MSCs. To investigate the biological function of A549 exosomes on adipogenic and osteogenic differentiation of AD-MSCs, $200 \mu \mathrm{g} / \mathrm{ml}$ A549-derived exosomes were added into AD-MSC culture medium. AD-MSCs treated with A549 exosomes were then cultured in osteogenic induction medium or adipogenic induction medium. A549 exosome treatment inhibited osteogenic differentiation, which was indicated by the decrease of ALP activity (Fig. 2A), as well as decreased mineral deposition detected by Alizarin red staining (Fig. 2B). Moreover, lower mRNA expression levels of osteo-specific markers were detected in exosome treated AD-MSCs (Fig. 2D). According to the results of Oil red $\mathrm{O}$ staining by day 10 of differentiation, the accumulation of lipid droplets decreased significantly after treatment with A549 exosomes (Fig. 2C). In addition,
Table I. Summary of microarray analysis results.

\begin{tabular}{lcc} 
Probe class & Total & $\begin{array}{r}\text { Differentially expressed } \\
\text { (fold change } \geq 2)\end{array}$ \\
\hline LncRNA & 30586 & $2775(9.1 \%)$ \\
mRNA & 26109 & $2439(9.3 \%)$ \\
Combined & 56695 & $5214(9.2 \%)$ \\
\hline
\end{tabular}

mRNA expression levels of adipogenic transcription factors and adipocyte-specific markers PPAR $\gamma$ and LPL decreased remarkably in cells treated with A549 exosomes compared with control group (Fig. 2E). The results above suggested that A549 acted as a negative regulator in osteogenic and adipogenic differentiation of AD-MSCs.

A549-derived exosomes did not induce fibroblastic differentiation of AD-MSCs. To determine whether or not lung tumor-derived exosomes could contribute to generation of TAFs from MSCs, we treated AD-MSCs with A549-derived exosomes. MSCs were cultured in medium containing $200 \mu \mathrm{g} / \mathrm{ml} \mathrm{A549-derived} \mathrm{exosomes} \mathrm{for} 6$ days. No morphological changes such as elongated cellular processes were observed (Fig. 3A). Additionally, we tested the expression of myofibroblastic/fibroblastic markers such as $\alpha$-SMA and FAP. FAP was increased in MSCs incubated with A549-derived exosomes compared to controls incubated in medium without exosomes after 6 days (Fig. 3B), but expression of $\alpha$-SMA was not changed. These results suggested that A549-derived exosomes did not induce fibroblastic differentiation of AD-MSCs, at least within 6 days. 
A
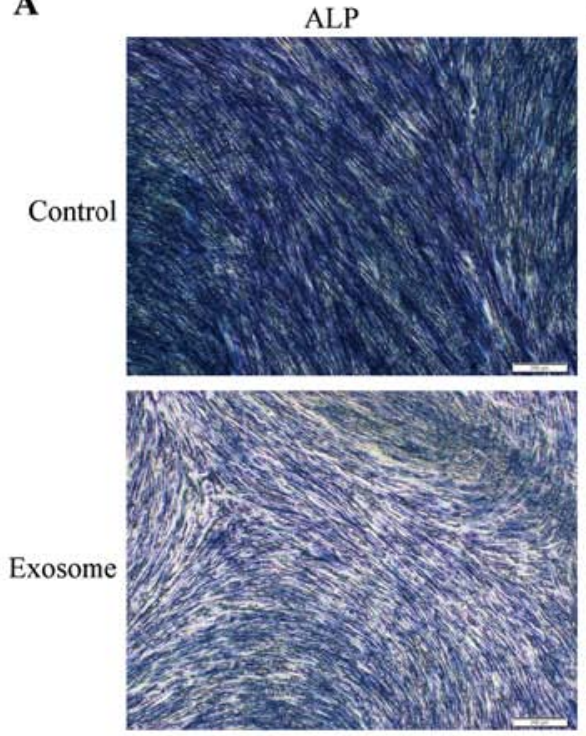

D

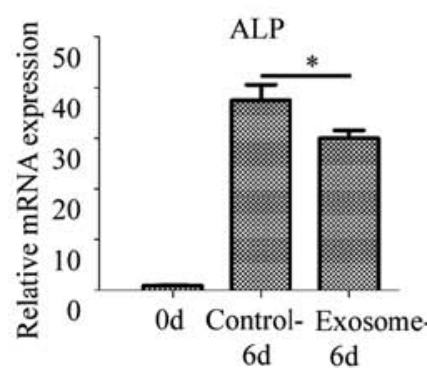

B
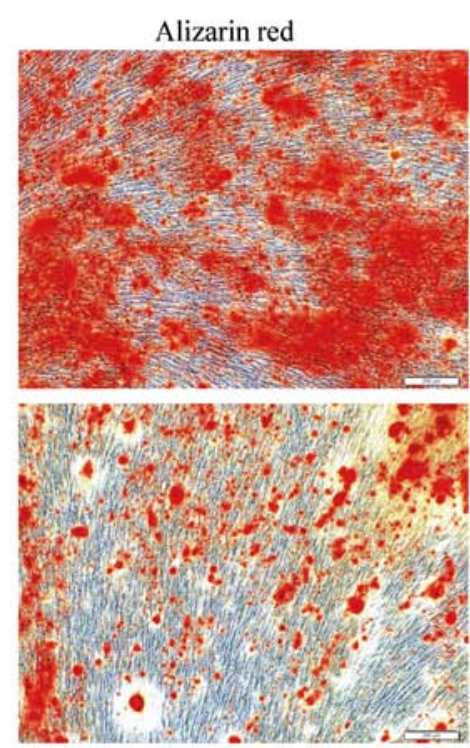

C
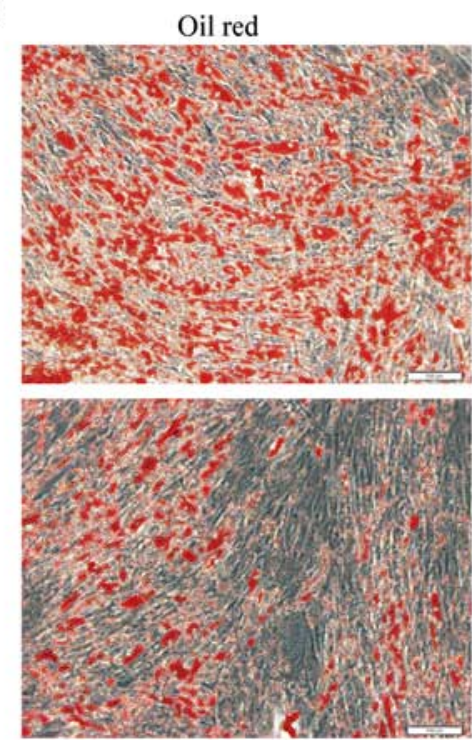

$\mathbf{E}$$$
\text { . }
$$
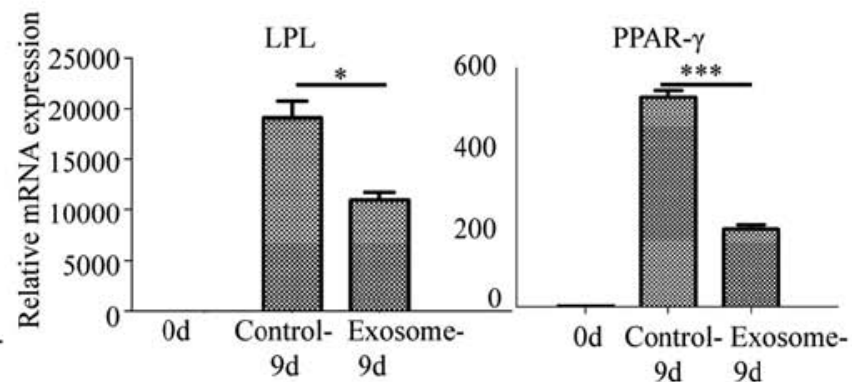

Figure 2. A549 exosomes inhibit osteogenic and adipogenic differentiation of AD-MSCs. (A) ALP staining at day 6 of osteogenic differentiation. (B) Alizarin red staining was performed to indicate the mineral deposition at day 12. (C) Oil Red O staining was performed at day 10 to visualize the lipid droplets accumulation in AD-MSCs. (D) Real-time PCR analysis of osteogenic specific genes (Runx 2 and ALP normalized to GAPDH). The data are the mean \pm SD of 3 independent experiments. ${ }^{*} \mathrm{P}<0.05$. (E) Real-time PCR analysis of adipogenic transcription factors (PPAR $\gamma$ ) and adipocyte-specific genes (LPL). The data, normalized to GAPDH, are the mean \pm SD of 3 independent experiments. ${ }^{*} \mathrm{P}<0.05$.

Overview of IncRNA profiles in A549 exosome-treated ADMSCs and control ADMSCs. To examine the lncRNA expression profiles in AD-MSCs treated with or without A549 exosomes, we used Arraystar Human LncRNA Microarray V3.0 which contains 30,586 lncRNA probes collected from Ref Seq, UCSC known genes and Gencode and 26,109 mRNA probes. Total RNA was extracted and examined for quality control before array. The OD260/OD280 ratios were approximately 2.0 , and the OD260/OD230 ratios were $>1.8$. The overview of lncRNA expression profiles is summarized in Table I and Fig. 4A and B. Overall, we found that $9.1 \%$ of lncRNAs (2775 out of 30586) and 9.3\% of protein-coding mRNA (2439 out of 26109) were differentially expressed (fold-change $\geq 2$; P-value $\leq 0.05$ ) between A549 exosome treated AD-MSCs and control AD-MSCs.

Fig. 4C shows the hierarchical cluster of lncRNAs expression between A549 exosome treated AD-MSCs and control AD-MSCs. Among the 2775 differentially expressed lncRNAs, 1263 lncRNAs were upregulated in experimental group compared to the control group, while 1512 lncRNAs were downregulated. The top 10 up- and down-regulated lncRNAs in A549 exosome treated AD-MSCs compared to control AD-MSCs are shown in Fig. 4D and Table II. We classified these differentially expressed lncRNAs into 5 groups:

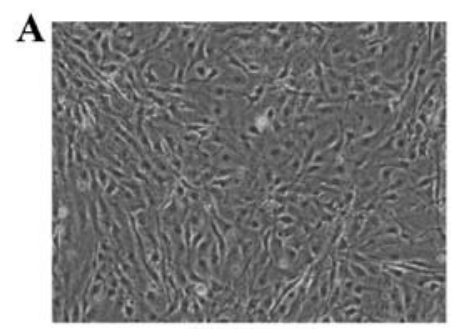

Exosome

B

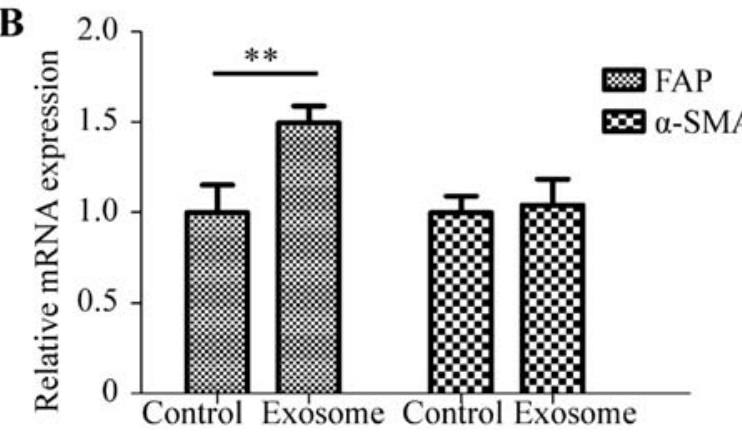

Figure 3. A549-derived exosomes did not induce fibroblastic differentiation of AD-MSCs. (A) Morphology of AD-MSCs was observed under a light microscope treated with or without exosome for 6 days. (B) Real-time PCR analysis of myofibroblastic/fibroblastic markers such as $\alpha$-SMA and FAP. ${ }^{*} \mathrm{P}<0.05$. 
A

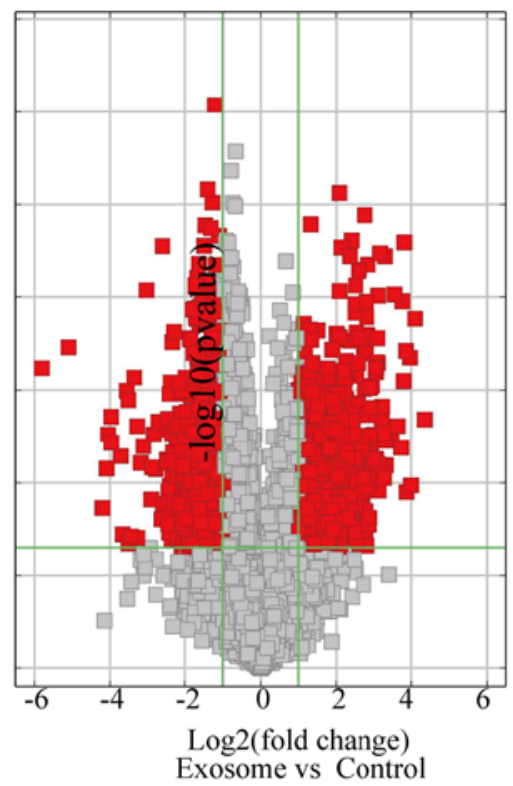

B

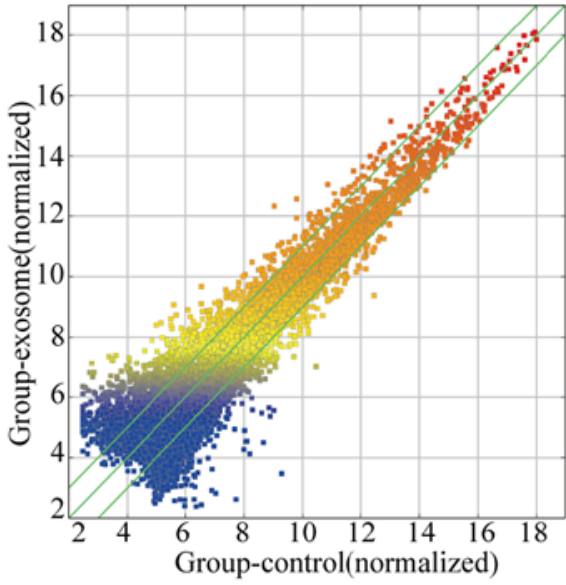

C

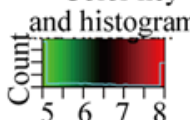

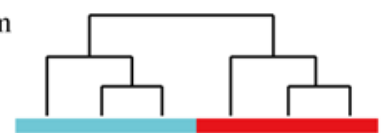

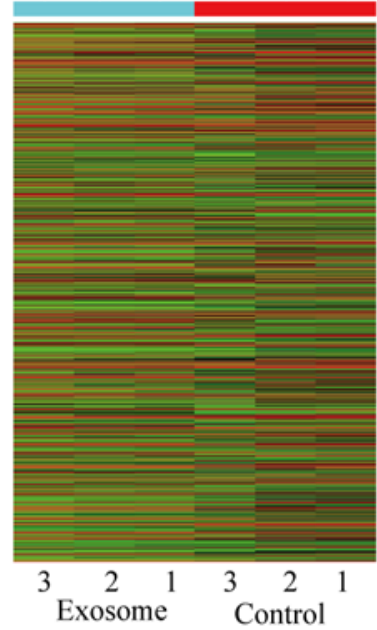

D

0.0

5.218055

7.3289194

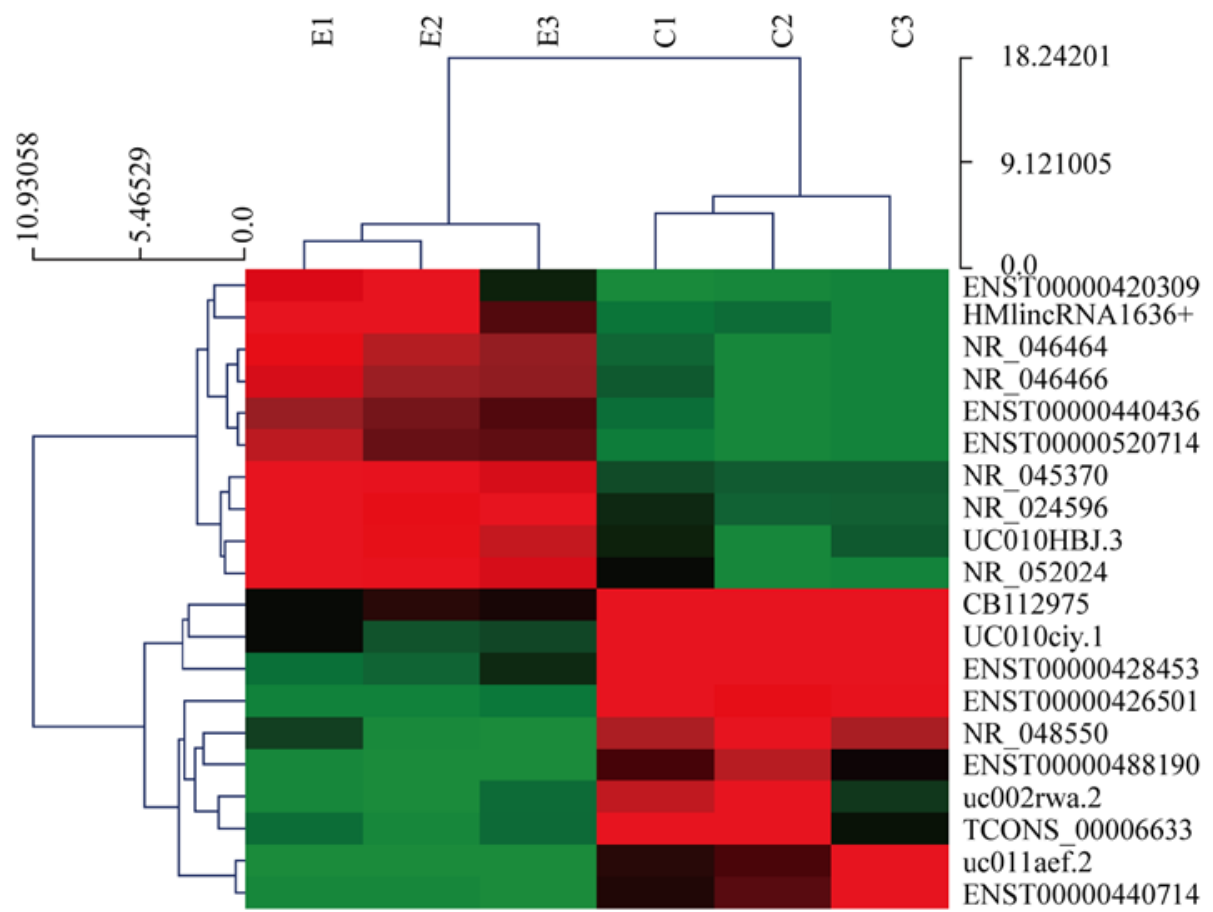

Figure 4. Overview of IncRNA profiles in A549 exosome treated AD-MSCs and control AD-MSCs. (A) Volcano plots of lncRNA expression profile. The vertical lines correspond to two-fold up and down and horizontal line represents a P-value of 0.05 . The red points in the plot represent differentially expressed lncRNAs with statistical significance. (B) The scatter plot is a visualization method used for assessing the lncRNA expression variations between A549 exosome treated AD-MSCs and control AD-MSCs. The values of the $\mathrm{X}$ and $\mathrm{Y}$ axes in the scatter plot are the averaged normalized signal values of the group ( $\log 2$ scale). The green lines are fold change lines (the default fold change given is 2). (C) hierarchical clustering of lnRNAs in A549 exosome treated AD-MSCs and control AD-MSCs. (D) The top 10 up- and down-regulated lncRNAs in A549 exosome treated AD-MSCs compared to control AD-MSCs (E represents exosome-treated MSCs, C represents control MSCs).

'sense-overlapping', the lncRNA exon is overlapping a coding transcript exon on the same genomic strand; 'intronic', the lncRNA is overlapping the intron of a coding transcript on the same genomic strand; 'antisense', the lncRNA is transcribed from the antisense strand; 'bidirectional', the lncRNA is oriented head to head to a coding transcript within $1000 \mathrm{bp}$; 'intergenic': there are no overlapping or bidirectional coding transcripts near the lncRNA.

Fig. 5A shows the distribution of the five classes of lncRNAs with changed expression in A549 exosome treated AD-MSCs. The lncRNAs are mainly between 200 and $3000 \mathrm{bp}$ in length. Fig. 5B shows the length distribution of 
Table II. The top 10 up- and down-regulated lncRNAs in A549 exosome treated AD-MSCs versus control AD-MSCs.

\begin{tabular}{|c|c|c|c|c|c|c|}
\hline Top 10 lncRNAs & $\begin{array}{l}\text { Chromosomal } \\
\text { localization }\end{array}$ & $\begin{array}{l}\text { RNA } \\
\text { length }\end{array}$ & Start locus & Start locus & $\begin{array}{l}\text { Associated } \\
\text { gene name }\end{array}$ & Relationship \\
\hline \multicolumn{7}{|l|}{ Upregulated } \\
\hline HMlincRNA1636+ & chrx & 8519 & 68199500 & 68208019 & & Intergenic \\
\hline NR_046464 & chr14 & 1687 & 101292444 & 101327360 & & Intergenic \\
\hline NR_052024 & $\operatorname{chr} 20$ & 803 & 33866708 & 33872520 & EIF6 & Sense-overlapping \\
\hline ENST00000420309 & chr2 & 573 & 70223961 & 70313407 & & Intergenic \\
\hline NR_046466 & chr14 & 1506 & 101292444 & 101327360 & & Intergenic \\
\hline NR_045370 & $\operatorname{chr} 20$ & 4760 & 62507483 & 62512243 & TPD52L2 & Sense-overlapping \\
\hline NR_024596 & $\operatorname{chr} 11$ & 1129 & 86014397 & 86056985 & C11 orf73 & Sense-overlapping \\
\hline ENST00000520714 & chr14 & 1351 & 101292454 & 101311828 & & Intergenic \\
\hline uc010hbj.3 & $\operatorname{chr} 22$ & 1172 & 51222224 & 51238065 & RABL2B & Bidirectional \\
\hline ENST00000440436 & $\operatorname{chr} 10$ & 480 & 6067940 & 6078390 & IL2RA & Antisense \\
\hline \multicolumn{7}{|l|}{ Downregulated } \\
\hline ENST00000428453 & $\operatorname{chr} 15$ & 4383 & 20588367 & 20711414 & & Intergenic \\
\hline ENST00000426501 & $\operatorname{chr} 15$ & 2874 & 20587868 & 20659133 & & Intergenic \\
\hline TCONS_00006633 & $\operatorname{chr} 3$ & 2229 & 125984828 & 125994041 & & Intergenic \\
\hline ENST00000440714 & chr21 & 292 & 40400460 & 40401053 & & Intergenic \\
\hline uc010ciy.1 & $\operatorname{chr} 16$ & 2079 & 89978911 & 89981576 & RP11-566K11.2 & Sense-overlapping \\
\hline NR_048550 & chr1 & 1604 & 218066241 & 218094146 & DSCR3 & Intergenic \\
\hline uc011aef.2 & $\operatorname{chr} 21$ & 458 & 38580954 & 38592893 & MSH2 & Antisense \\
\hline uc002rwa.2 & $\operatorname{chr} 2$ & 2000 & 47713159 & 47715691 & CPA3 & Antisense \\
\hline ENST00000488190 & $\operatorname{chr} 3$ & 1414 & 148568719 & 148677899 & & Antisense \\
\hline CB112975 & $\operatorname{chr} 13$ & 379 & 30229248 & 30229615 & & Intergenic \\
\hline
\end{tabular}

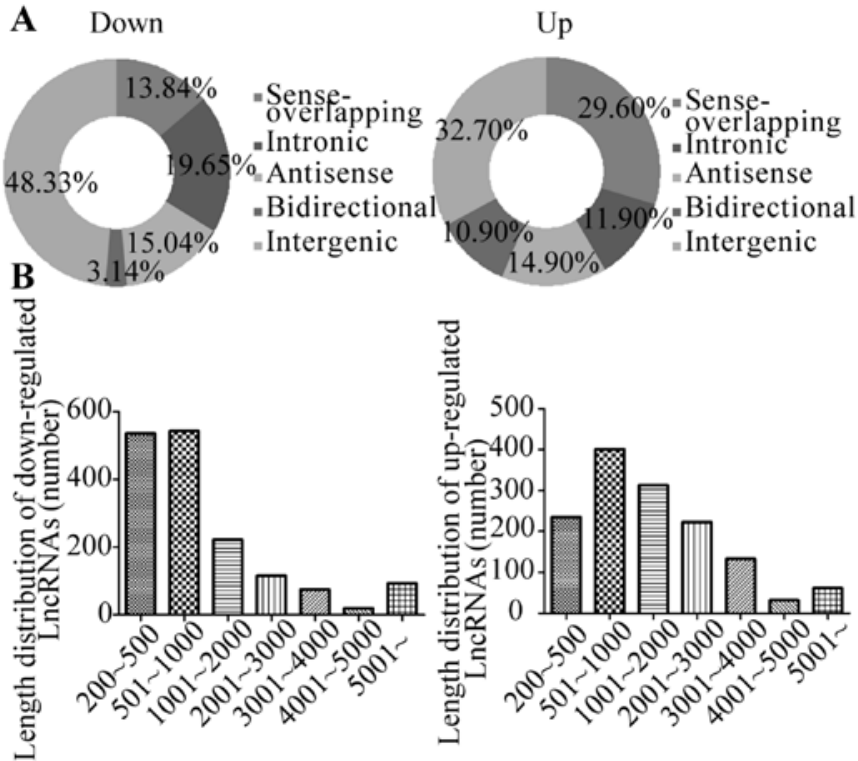

Figure 5. Characteristics of lncRNAs with changed expression in A549 exosome treated AD-MSCs compared to control AD-MSCs. (A) Distribution of five classes of differentially expressed lncRNAs. (B) Length distribution of differentially expressed lncRNAs.

differentially expressed lncRNAs. The majority of the differentially expressed lncRNAs have a length between 500 and $1000 \mathrm{bp}$.
GO and pathway analysis of differentially expressed genes in AD-MSCs treated with A549 exosomes. The microarray also detected 2439 differentially expressed mRNA (absolute fold-change $\geq 2$; P-value $\leq 0.05$ ). Among them, 1940 mRNAs were upregulated in AD-MSCs treated with A549 exosomes compared to the control group, while 499 mRNAs were downregulated. Fig. 6A showed the hierarchical cluster of mRNA expression between A549 exosome treated AD-MSCs and control AD-MSCs. Specifically, we picked out the top 10 up- and down-regulated mRNAs. (Fig. 6B). Moreover, we performed $\mathrm{GO}$ analysis to determine the gene and gene product enrichment in biological processes, cellular components and molecular functions. We found that the highest enriched GOs targeted by upregulated mRNAs in A549 exosome treated AD-MSCs were mRNA metabolic process (ontology: biological process) (Fig. 7A), intracellular part (ontology: cellular component) (Fig. 7B) and structural constituent of ribosome (ontology: molecular function) (Fig. 7C). The highest enriched GOs targeted by the downregulated transcripts in A549 exosome treated AD-MSCs were detection of mechanical stimulus involved in sensory perception (ontology: biological process) (Fig. 7D), primary cilium (ontology: cellular component) (Fig. 7E) and acetylgalactosaminyltransferase activity (ontology: molecular function) (Fig. 7F). Pathway analysis indicated that 32 pathways were upregulated and 7 were downregulated in A549 exosome treated MSCs. Fig. 7G and $\mathrm{H}$ show the top 10 of the upregulated and 7 of the downregulated pathways, respectively. 
A
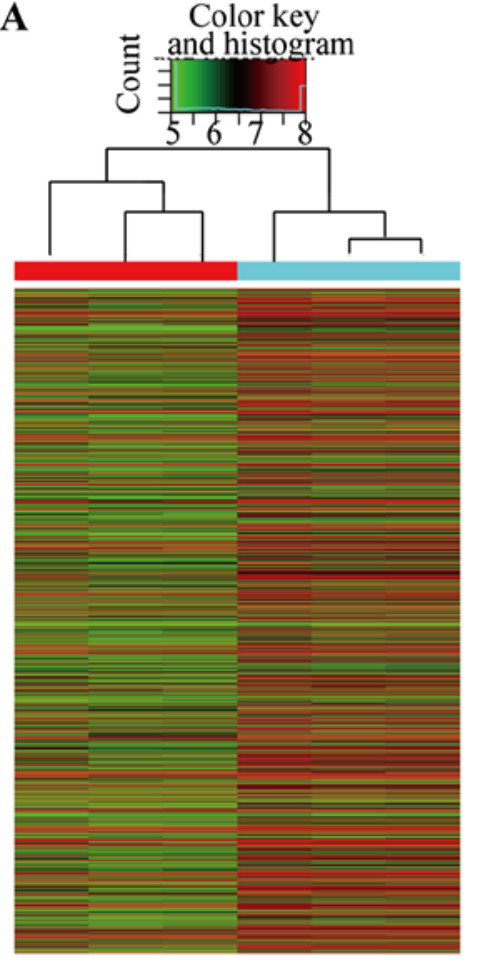

Control
B

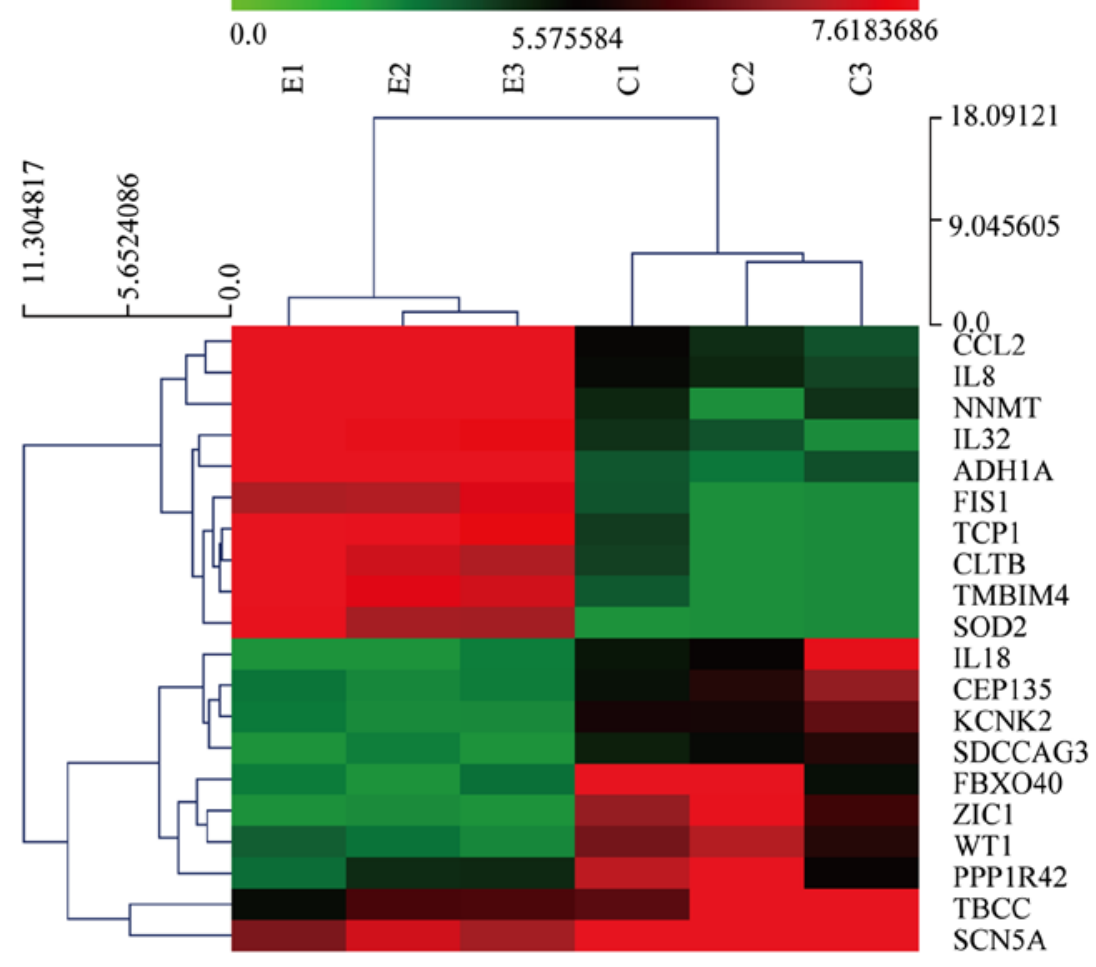

Figure 6. Overview of mRNA profiles in A549 exosome treated AD-MSCs and control AD-MSCs. (A) Hierarchical clustering of 1nRNAs in A549 exosome treated AD-MSCs and control AD-MSCs. (B) The top 10 up- and down-regulated mRNAs in A549 exosome treated AD-MSCs compared to control AD-MSCs.

\section{Discussion}

Interactions between tumor cells and other cell components in the tumor microenvironment play profound roles in driving tumor initiation and progression. Recently, accumulating evidence provides clues for the involvement of exosomes in mediating such interactions (20-22). Tumor derived exosomes usually carry tumor antigens which make them useful as powerful anticancer vaccines (23). They also play active roles in various biological behaviors of tumor cells such as immune modulation and drug resistance. For instance, breast cancer-derived exosomes are capable of inducing an inflammatory response in macrophages, which may ultimately result in an enhanced rate of metastatic tumor development (24). Xiao et al found A549 cells exosomes are involved in the decrease of the sensitivity of A549 cells to DDP (25).

Tumor exosomes cause a myriad of biological changes in target cells with the transfer of mRNAs or proteins. Our study demonstrated that lung tumor cell A549 derived exosomes could inhibit osteogenic and adipogenic differentiation of AD-MSCs. MSCs may support tumor propagation or dissemination by preventing recognition of the tumor cells by the immune system or by promoting tumor cell invasiveness $(26,27)$. Cross-talk between MSCs within tumor stroma and cancer cells has been identified to contribute to tumor progression and metastasis through stromal formation or modulation of cell proliferation. Recent studies demonstrated that tumor-derived exosomes can function in mediating such cross-talk between MSCs and cancer cells. However, the precise mechanisms by which tumor exosomes affect MSCs remain largely unknown.
Specifically, whether epigenetic regulators such as long non-coding RNA are involved in this process remains unclear. Long non-coding RNAs have been established to participate in various biological processes that are crucial for development and differentiation. Although the vast majority of their functions remain unexplored, there is evidence that some lncRNAs are involved in regulating stem cell properties. Herein, a major focus of our study was to define the repertoire of lncRNAs in tumor exosome treated AD-MSCs.

To our knowledge, this is the first study to give a comprehensive overview of the IncRNA transcriptome changes in MSCs after stimulation with tumor derived exosomes. We found $9.1 \%$ of lncRNAs (2775 out of 30586) and 9.3\% of protein-coding mRNA (2439 out of 26109) were differentially expressed (fold-change $\geq 2$; P-value $\leq 0.05$ ) in lung tumor cell exosome treated MSCs. Furthermore, we characterized the differentially expressed IncRNAs through their classes and length distribution and correlated them with differentially expressed mRNA. Noteworthy, GO analysis of biological process showed that upregulated mRNAs were enriched in mRNA metabolic process, while downregulated ones were enriched in detection of mechanical stimulus involved in sensory perception. Pathway analysis indicated that 32 pathways were upregulated while 7 were downregulated in A549 exosome treated MSCs. These results suggest that tumor exosomes could stimulate MSCs into an active state.

In summary, our study is the first to demonstrate that a set of lncRNAs is significantly regulated in AD-MSCs upon treatment with tumor derived exosomes, suggesting a role of IncRNAs in the regulation of AD-MSCs. This will provide some new insights into the involvement of IncRNAs in 
A

Protein localization to endoplasmic reticulum Cellular cataolic process Translational elongation Electron transport chain Cellular metabolic process

Establishment of protein localization to organella Cellular respiration Cellular macromolecule catabolic process Respiratory electron transport chain
mRNA metabolic process Enrichment score (-log10(pvalue))

B

Intracellular organelle part Membrane-bounded organelle Intracellular membrane-bounded Organelle Intracellular organelle Mitochondrion IntracellularCytoplasmic partIntracellular part

Enrichment score (-log10(pvalue))

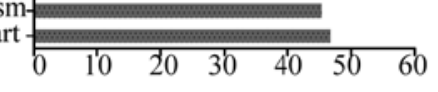

D

Sensory perception of mechanical stimulus

Regulation of transcription

Chromosome segregation Transcription

Regulation of biomineral tissue development

Meiotic chromosome segregation

Regulation of RNA biosynthetic process Actin-mediated cell contraction Detection of mechanical stimulus 25

E
Enrichment score (-log10(pvalue))

C

\section{F}

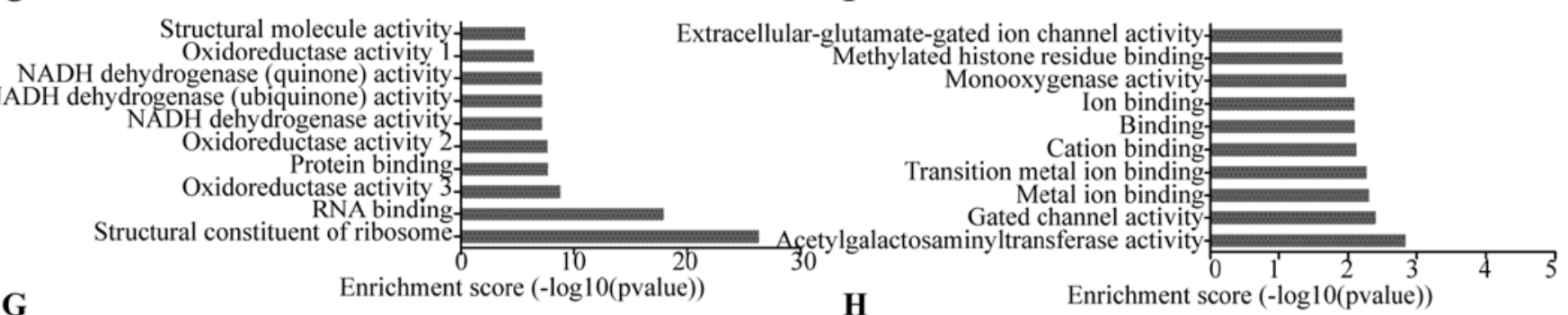

G

Sulfur metabolism

Protein processing in endoplasmic reticulum Proteasome

Non-alcoholic fatty liver disease (NAFLD) Spliceosome

Alzheimer's disease

Parkinson's disease

Oxidative phosphorylation

Huntington's disease Ribosome

Enrichment
Starch and sucrose metabolism Mucin type O-Glygan biosythesis

Olfactory transduction

Drug metabolism-cytochrome P450

Carbohydrate digestion and absorbtion

Ras signalling pathway Steroid hormone biosynthesis

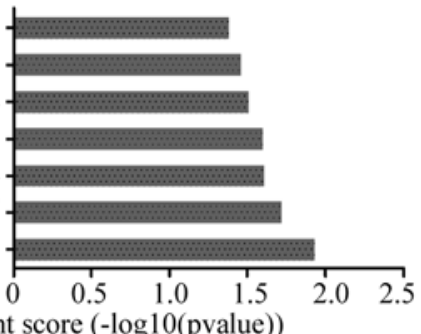

Figure 7. GO term enrichment and pathway analysis of the differentially expressed genes. (A-C) genes upregulated in exosome treated AD-MSCs. (D-F) Genes downregulated in exosome treated AD-MSCs. (G) Top 10 of the upregulated pathways in exosome treated AD-MSCs. (H) Seven of the downregulated pathways in exosome treated AD-MSCs.

exosome-mediated crosstalk between MSCs and tumor cells, which is a novel mechanism whereby tumor cells educate MSCs to modulate the tumor microenvironment.

\section{Acknowledgements}

This study was supported by grants from National Natural Science Foundation of China (no. 30830052, 30700321, 30800429) and Program for Cheung Kong Scholars and Innovative Research Team in University-PCSIRT (no. IRT0909).

\section{References}

1. Quante M, Tu SP, Tomita H, Gonda T, Wang SS, Takashi S, Baik GH, Shibata W, Diprete B, Betz KS, et al: Bone marrowderived myofibroblasts contribute to the mesenchymal stem cell niche and promote tumor growth. Cancer Cell 19: 257-272, 2011.

2. Joyce JA and Pollard JW: Microenvironmental regulation of metastasis. Nat Rev Cancer 9: 239-252, 2009.
3. Bhowmick NA and Moses HL: Tumor-stroma interactions. Curr Opin Genet Dev 15: 97-101, 2005.

4. Pietras K and Ostman A: Hallmarks of cancer: Interactions with the tumor stroma. Exp Cell Res 316: 1324-1331, 2010.

5. Zhu W, Xu W, Jiang R, Qian H, Chen M, Hu J, Cao W, Han C and Chen Y: Mesenchymal stem cells derived from bone marrow favor tumor cell growth in vivo. Exp Mol Pathol 80: 267-274, 2006.

6. Karnoub AE, Dash AB, Vo AP, Sullivan A, Brooks MW, Bell GW, Richardson AL, Polyak K, Tubo R and Weinberg RA: Mesenchymal stem cells within tumour stroma promote breast cancer metastasis. Nature 449: 557-563, 2007.

7. Pittenger MF, Mackay AM, Beck SC, Jaiswal RK, Douglas R, Mosca JD, Moorman MA, Simonetti DW, Craig S and Marshak DR: Multilineage potential of adult human mesenchymal stem cells. Science 284: 143-147, 1999.

8. Roorda BD, ter Elst A, Kamps WA and de Bont ES: Bone marrow-derived cells and tumor growth: Contribution of bone marrow-derived cells to tumor micro-environments with special focus on mesenchymal stem cells. Crit Rev Oncol Hematol 69: 187-198, 2009.

9. Ye J, Wu D, Wu P, Chen Z and Huang J: The cancer stem cell niche: Cross talk between cancer stem cells and their microenvironment. Tumour Biol 35: 3945-3951, 2014. 
10. Egeblad M, Nakasone ES and Werb Z: Tumors as organs: Complex tissues that interface with the entire organism. Dev Cell 18: 884-901, 2010.

11. Grange C, Tapparo M, Collino F, Vitillo L, Damasco C, Deregibus MC, Tetta C, Bussolati B and Camussi G: Microvesicles released from human renal cancer stem cells stimulate angiogenesis and formation of lung premetastatic niche. Cancer Res 71: 5346-5356, 2011

12. Chen WX, Cai YQ, Lv MM, Chen L, Zhong SL, Ma TF, Zhao JH and Tang JH: Exosomes from docetaxel-resistant breast cancer cells alter chemosensitivity by delivering microRNAs. Tumour Biol 35: 9649-9659, 2014.

13. Iero M, Valenti R, Huber V, Filipazzi P, Parmiani G, Fais S and Rivoltini L: Tumour-released exosomes and their implications in cancer immunity. Cell Death Differ 15: 80-88, 2008.

14. Chowdhury R, Webber JP, Gurney M, Mason MD, Tabi Z and Clayton A: Cancer exosomes trigger mesenchymal stem cell differentiation into pro-angiogenic and pro-invasive myofibroblasts. Oncotarget 6: 715-731, 2015.

15. Haga H, Yan IK, Takahashi K, Wood J, Zubair A and Patel T: Tumour cell-derived extracellular vesicles interact with mesenchymal stem cells to modulate the microenvironment and enhance cholangiocarcinoma growth. J Extracell Vesicles 4: 24900, 2015

16. Yang Y, Bucan V, Baehre H, von der Ohe J, Otte A and Hass R: Acquisition of new tumor cell properties by MSC-derived exosomes. Int J Oncol 47: 244-252, 2015.

17. Cao Y, Sun Z, Liao L, Meng Y, Han Q and Zhao RC: Human adipose tissue-derived stem cells differentiate into endothelial cells in vitro and improve postnatal neovascularization in vivo. Biochem Biophys Res Commun 332: 370-379, 2005.

18. Ohshima K, Inoue K, Fujiwara A, Hatakeyama K, Kanto K, Watanabe Y, Muramatsu K, Fukuda Y, Ogura S, Yamaguchi K, et al: Let-7 microRNA family is selectively secreted into the extracellular environment via exosomes in a metastatic gastric cancer cell line. PLoS One 5: e13247, 2010.
19. Hood JL, Pan H, Lanza GM and Wickline SA; Consortium for Translational Research in Advanced Imaging and Nanomedicine (C-TRAIN): Paracrine induction of endothelium by tumor exosomes. Lab Invest 89: 1317-1328, 2009.

20. Boelens MC, Wu TJ, Nabet BY, Xu B, Qiu Y, Yoon T, Azzam DJ, Twyman-Saint Victor C, Wiemann BZ, Ishwaran $\mathrm{H}$, et al: Exosome transfer from stromal to breast cancer cells regulates therapy resistance pathways. Cell 159: 499-513, 2014.

21. Zhang X, Yuan X, Shi H, Wu L, Qian H and Xu W: Exosomes in cancer: Small particle, big player. J Hematol Oncol 8: 83, 2015.

22. Tang MK and Wong AS: Exosomes: Emerging biomarkers and targets for ovarian cancer. Cancer Lett 367: 26-33, 2015.

23. Cho JA, Yeo DJ, Son HY, Kim HW, Jung DS, Ko JK, Koh JS, Kim YN and Kim CW: Exosomes: A new delivery system for tumor antigens in cancer immunotherapy. Int J Cancer 114: 613-622, 2005.

24. Chow A, Zhou W, Liu L, Fong MY, Champer J, Van Haute D, Chin AR, Ren X, Gugiu BG, Meng Z, et al: Macrophage immunomodulation by breast cancer-derived exosomes requires Toll-like receptor 2-mediated activation of NF- $\kappa$ B. Sci Rep 4: 5750, 2014.

25. Xiao X, Yu S, Li S, Wu J, Ma R, Cao H, Zhu Y and Feng J. Exosomes: Decreased sensitivity of lung cancer A549 cells to cisplatin. PLoS One 9: e89534, 2014.

26. Ohno S, Tachibana M, Fujii T, Ueda S, Kubota H and Nagasue N: Role of stromal collagen in immunomodulation and prognosis of advanced gastric carcinoma. Int J Cancer 97: 770-774, 2002.

27. Keleg S, Büchler P, Ludwig R, Büchler MW and Friess H: Invasion and metastasis in pancreatic cancer. Mol Cancer 2: 14, 2003. 